\title{
World Students Ultrasound Conference (WStUC)
}

World Federation for Ultrasound in Medicine and Biology had a premiere this year by organizing a series of Ultrasound Webinars dedicated for students and young doctors. The project was initiated by Prof. D. Nurnberg - WFUMB Student Education Task Force 2019-2021 as a substitute for the usual ultrasound training in pandemic times. It consisted in a series of webinars where renowned physicians and researchers in the field of ultrasound were invited to hold their presentation in a more "student friendly" manner. The project was of notably success and raised a lot of interest among students, thus opening the door towards new projects in student ultrasound teaching.

Next year between $25-28^{\text {th }}$ of May, Timisoara (Romania) will be the "The World Capital of Ultrasound", organizing three prestigious ultrasound events: The $18^{\text {th }}$ World Federation for Medicine and Biology Congress - WFUMB 2021, the $33^{\text {rd }}$ Congress of EFSUMB - EUROSON 2021 and the $25^{\text {th }}$ National Conference of The Romanian Society of Ultrasound in Medicine and Biology. With so many ultrasound events taking place in one city, the idea of organizing the first World Ultrasound Conference for Students (WStUC-Students Ultrasound Conference, endorsed by WFUMB) was a legitimate one.

After more than one year during which the online interaction prevailed, WStUC will be a great opportunity to resume the ultrasound synergy and communication. The conference will be centered mainly on students and young doctors who are discovering the importance of ultrasound. Lectures form basic to advance, hands on stations, quizzes and meet the expert's session will play the part of the program. Students Ultrasound Educa- tion will be also an important topic of the meeting, through which different concepts and dedicated curriculums will be discussed. Participants will also have the convenience to present their work as a poster or oral presentation in dedicated sessions.

The congress will be held in Timisoara, a strong university center and a multicultural city that will be the "European Capital of Culture" in 2023, at the Regional Business Center.

See you in May 2022 in Timișoara!

WStUC Organizing committee Tudor Moga MD

Prof. Dieter Nürnberg

More details can be found on the congress website www.wfumb2022.com 